\title{
Midgut immune responses induced by bacterial infection in the silkworm, Bombyx mori*
}

\author{
Lei ZHANG, Yan-wen WANG, Zhi-qiang LU ${ }^{\dagger *}$ \\ (Key Laboratory of Plant Protection Resources and Pest Management, Ministry of Education, \\ Northwest A\&F University, Yangling 712100, China) \\ †E-mail: zhiqiang.lu@nwsuaf.edu.cn \\ Received Mar. 12, 2015; Revision accepted July 6, 2015; Crosschecked Sept. 17, 2015
}

\begin{abstract}
Insect gut epithelial cells produce reactive oxygen species (ROS) and antimicrobial peptides (AMPs) to protect hosts from pathogenic microorganisms. In this study, we evaluate the pathogenicity of Pseudomonas aeruginosa and Bacillus bombysepticus in the silkworm, Bombyx mori. Survival curves show that B. bombysepticus is deadly when larval silkworms are infected orally. Bacterial infection caused intestinal hydrogen peroxide $\left(\mathrm{H}_{2} \mathrm{O}_{2}\right)$ and nitric oxide (NO) levels to increase significantly by 8 and $16 \mathrm{~h}$ post-infection (hpi), respectively. Real-time quantitative polymerase chain reaction (qPCR) analysis shows that the transcription levels of dual oxidase (Duox) and catalase $(C A T)$ are highly up-regulated by $P$. aeruginosa infection at $8 \mathrm{hpi}$. $P$. aeruginosa infection induced nitric oxide synthase 2 (NOS2) expression at $16 \mathrm{hpi}$, which contributes to the generation of NO. mRNA levels of AMP genes, specifically Glovorin 2 and Glovorin 3, which obviously increase during the early infection stage. These results indicate that invading bacteria elevate intestinal ROS and NO levels and induce AMP gene transcription, which contributes to intestinal immune defense.
\end{abstract}

Key words: Bombyx mori, Midgut, Immune, Hydrogen peroxide, Nitric oxide, Antimicrobial peptide doi: 10.1631 jzus.B1500060

Document code: A

CLC number: S884.4

\section{Introduction}

Innate immunity, which exists in all metazoan organisms, is an evolutionarily conserved system for defending the host against microbial invasion. In Drosophila, the gut epithelium is the first line of protection for the host against microorganismal invasion and proliferation (Hoffmann and Reichhart, 2002). Two types of immune molecules are involved in Drosophila gut defense. First, the production of reactive oxygen species (ROS) and nitric oxide (NO) was demonstrated to kill pathogens in the gut epithe-

\footnotetext{
Corresponding author

* Project supported by the National Natural Science Foundation of China (No. 31272497)

(1) ORCID: Zhi-qiang LU, http://orcid.org/0000-0002-7803-8442

(C) Zhejiang University and Springer-Verlag Berlin Heidelberg 2015
}

lium and to trigger downstream immune responses (Wink et al., 2011). Erwinia carotovora carotovora 15 (Ecc15) infection increases the levels of ROS synthesized by dual oxidase (Duox) in the Drosophila gut. Duox-RNA interference (RNAi) flies showed increased mortality and failed to control Ecc 15 proliferation in the gut, suggesting that Duox is the main enzyme inducing ROS during gut infection (Ha et al., 2005b). In Aedes aegypti, midgut epithelial cells generate ROS to control bacterial growth (Oliveira et al., 2011). Excess ROS is toxic to the host and is degraded by immune responsive catalase (IRC) to maintain homeostatic redox balance. IRC-RNAi flies exhibited ROS over-production and increased lethality, indicating that IRC plays an antioxidant role in the host defense system (Ha et al., 2005a). NO is generated by nitric oxide synthase (NOS) enzymes, 
including NOS1, NOS2, and NOS3. NOS2 is inducible, while the other two are constitutively expressed (Wink et al., 2011). Lipopolysaccharide (LPS) stimulation induced the expression of NOS in Bombyx mori (Imamura et al., 2002).

The second intestinal immune defense is the generation of local antimicrobial peptides (AMPs) via the immune deficiency (IMD) pathway (Tzou et al., 2000). Drosomycin and Diptericin are induced in the gut of Drosophila after Erwinia carotovora infection (Basset et al., 2000). In B. mori, local AMP genes, including Cecropin A1 (CecA1), Gloverin 1 (Glov1), Glov2, Glov3, Glov4, and lysozyme (Lys), are induced by Staphylococcus aureus, whereas the expression of CecA1, Glov3 and Glov4 is sometimes inhibited by Escherichia coli infection (Wu et al., 2010b).

In this study, we demonstrate that intestinal hydrogen peroxide $\left(\mathrm{H}_{2} \mathrm{O}_{2}\right)$ and $\mathrm{NO}$ levels are elevated after bacterial infection and that the mRNA transcription levels of ROS-related genes and AMP genes are also up-regulated. These results indicate that ROS and AMP have vital defense roles in the midgut of silkworms.

\section{Materials and methods}

\subsection{Silkworm rearing}

Silkworm larvae (Nistari strain) were reared on mulberry leaves at $27^{\circ} \mathrm{C}, 70 \%$ relative humidity, and a 12-h light:12-h dark photoperiod.

\subsection{Oral infection}

Pseudomonas aeruginosa and Bacillus bombysepticus were cultured overnight in Luria-Bertani (LB) medium at $37{ }^{\circ} \mathrm{C}$. The bacterial pellet was collected by centrifugation at $8000 \mathrm{~g}$ for $15 \mathrm{~min}$ and washed three times with $0.85 \%(8.5 \mathrm{~g} / \mathrm{L}) \mathrm{NaCl}$. The harvested bacterial cells were suspended in $400 \mu 10.85 \% \mathrm{NaCl}$ to an optical density at $600 \mathrm{~nm}\left(\mathrm{OD}_{600 \mathrm{~nm}}\right)$ of 40 and used for silkworm oral infection. Fresh mulberry leaves were cut into $1 \mathrm{~cm} \times 1 \mathrm{~cm}$ pieces and coated with bacterial suspensions. Day 3 fifth instar larvae were starved for $12 \mathrm{~h}$ before feeding them bacteria. A group of 20 larvae were used for oral infection. Each larva was fed $20 \mu \mathrm{l}$ bacteria or $0.85 \% \mathrm{NaCl}$ as a control. Midguts were collected at different time points (4, 8,16 , and $24 \mathrm{~h}$ ) after feeding.

\subsection{Mortality recording and colony forming unit (CFU) assay}

A group of 20 larvae were infected as described above to evaluate mortality. The number of surviving larvae was recorded every $24 \mathrm{~h}$. Another nine larvae were infected for a bacterial persistence assay. At 0.5 , 12 , and $24 \mathrm{~h}$ post-infection, larvae were dissected, and the peritrophic membranes and their contents (PMC) were collected. Gut contents from individual larvae were separated from the PMC by centrifugation at $500 \mathrm{~g}$ for $10 \mathrm{~min}$. The supernatant was diluted 100-fold with fresh LB and incubated on a LB agar plate with ampicillin $(100 \mu \mathrm{g} / \mathrm{ml})$ at $37{ }^{\circ} \mathrm{C}$ for $12 \mathrm{~h}$. The numbers of colony forming units (CFUs) were counted. Three larvae were selected for each time point, and the experiment was repeated three times.

\section{$2.4 \mathrm{H}_{2} \mathrm{O}_{2}$ level measurement in the midgut}

After oral infection, five larvae were dissected to collect the PMC at different time points. Gut contents were separated from the PMC by centrifugation at $13000 \mathrm{~g}$ for $10 \mathrm{~min}$. The supernatants were transferred to Amicon Ultra 10K filters (Millipore, Billerica, MA, USA) and centrifuged at $13000 \mathrm{~g}$ for 5 min. The flow-through samples were used in $\mathrm{H}_{2} \mathrm{O}_{2}$ assays using the Amplex Red Hydrogen Peroxide/ Peroxidase Assay Kit (Invitrogen, Carlsbad, CA, USA) according to the manufacturer's protocol.

\subsection{Gene expression analysis using real-time quantitative PCR}

Real-time quantitative polymerase chain reaction (qPCR) was used to evaluate the expression levels of ROS-related genes and AMP genes. Total RNA was extracted from the midguts of silkworms after various treatments and purified using the Direct-zol ${ }^{\mathrm{TM}}$ RNA MiniPrep Kit (Zymo, Irvine, CA, USA). First strand complementary DNA (cDNA) was synthesized using SuperScript III reverse transcriptase (Invitrogen, Carlsbad, CA, USA) following the manufacturer's instructions. B. mori initiation factor $4 \alpha(I F 4 \alpha)$ was used as an internal control to normalize the expression of target genes (Wu et al., 2010a). All specific primers for qPCR are listed in Table 1. qPCR was performed using a FastStart Essential DNA Green Master mix (Roche, Indianapolis, IN, USA) with the CFX96 Real-Time PCR Detection System (Bio-Rad, Hercules, California, USA). qPCR was performed 
using an initial denaturation at $95{ }^{\circ} \mathrm{C}$ for $10 \mathrm{~min}$, followed by 39 cycles of amplification $\left(95^{\circ} \mathrm{C}\right.$ for $10 \mathrm{~s}$, $55^{\circ} \mathrm{C}$ for $20 \mathrm{~s}$, and $72{ }^{\circ} \mathrm{C}$ for $30 \mathrm{~s}$ ), and the melting curve analysis was performed from 65 to $95{ }^{\circ} \mathrm{C}$. The relative expression levels of target genes were analyzed using the $2^{-\Delta \Delta C_{\mathrm{T}}}$ method (Livak and Schmittgen, 2001). All experiments were repeated independently three times.

Table 1 Primers for qPCR

\begin{tabular}{cl}
\hline Gene & \multicolumn{1}{c}{ Primer sequence } \\
\hline Duox & F: 5' GCTTCGTCGTATAACTCTGTGG 3' \\
& R: 5' TGCAGGGTGGAAGTTTGG 3' \\
CAT & F: 5' GGGAGCGTATTCCAGAAC 3' \\
& R: 5' GAGGGTCACGAACAGTATCA 3' \\
NOS1 & F: 5' AGTTGGCTTGGCGTAATG 3' \\
& R: 5' TACCGTCTGTGCGTTGTG 3' \\
NOS2 & F: 5' CGGGAAAGACCCTGACTA 3' \\
& R: 5' AAACGCATACTGGAGACG 3' \\
Att2 & F: 5' TTCAAACAGAAGGTGGGC 3' \\
& R: 5' GACGGAGATTGGAACAGG 3' \\
CecB6 & F: 5' TCCTTCGTCTTCGCTCTG 3' \\
& R: 5' GATGCCGTCACGGATGTT 3' \\
CecD & F: 5' CTCCCGGCAACTTCTTCA 3' \\
& R: 5' CGAACCCTCTGACCCATT 3' \\
Glov2 & F: 5' ACGGACCTTCTGATTACGC 3' \\
& R: 5' CATTCTTGTTCGCCCAGT 3' \\
Glov3 & F: 5' GACACGAGAATGGGAGGAG 3' \\
& R: 5' AAGACCCTGGTGCCGTAA 3' \\
Mor & F: 5' CAAGGCCATTAAGACTGT 3' \\
& R: 5' TTTCTTTTCTTCGGTTTC 3' \\
\hline
\end{tabular}

F: forward; R: reverse

\subsection{Statistical analysis}

All data are presented as mean \pm SD. The unpaired Student's $t$-test was used to compare expression differences between control and infection conditions. Bonferroni's correction was used to determine the critical significance level. The log-rank test was used to analyze the survival rate using GraphPad Prism 5 (GraphPad Software, Inc., La Jolla, CA, USA).

\section{Results}

\subsection{B. bombysepticus infection in the silkworm}

When silkworms were infected with $P$. aeruginosa or B. bombysepticus, death rarely occurred during the first $5 \mathrm{~d}$. Afterwards, the survival rate after $B$. bombysepticus infection was reduced remarkably, reaching approximately $20 \%$ by Day 8 , while only
$10 \%$ mortality was seen by Day 8 after $P$. aeruginosa infection (Fig. 1). These results suggest that $B$. bombysepticus is more pathogenic to silkworms.

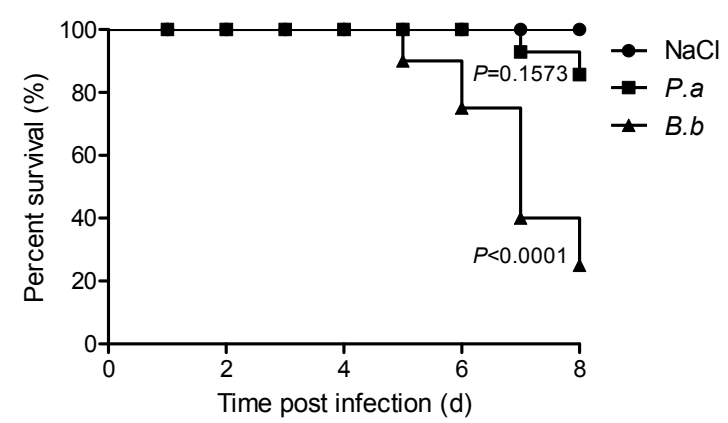

Fig. 1 Survival rate of silkworm larvae after bacterial feeding

NaCl: control; P.a: Pseudomonas aeruginosa; B.b: Bacillus bombyseptieus. Twenty larvae were used for each treatment

\subsection{Bacterial numbers in infected larvae}

To investigate the persistence of ingested bacteria in infected larvae, we examined the viability of bacteria in the gut. At $0.5 \mathrm{~h}$ post oral infection, compared with the control, $P$. aeruginosa was more persistent, at 100-fold CFUs higher, than B. bombysepticus, but by $12 \mathrm{~h}$ and $24 \mathrm{~h}$, no $P$. aeruginosa cells were growing on the plates. In contrast, $B$. bombysepticus persisted and proliferated in the gut with CFUs increasing gradually and reaching a maximum at $24 \mathrm{~h}$ (Fig. 2). These findings suggest that the silkworm employs different mechanisms to combat different invading bacteria. Alternatively, B. bombysepticus and $P$. aeruginosa may have different abilities to strive against silkworm immune responses.

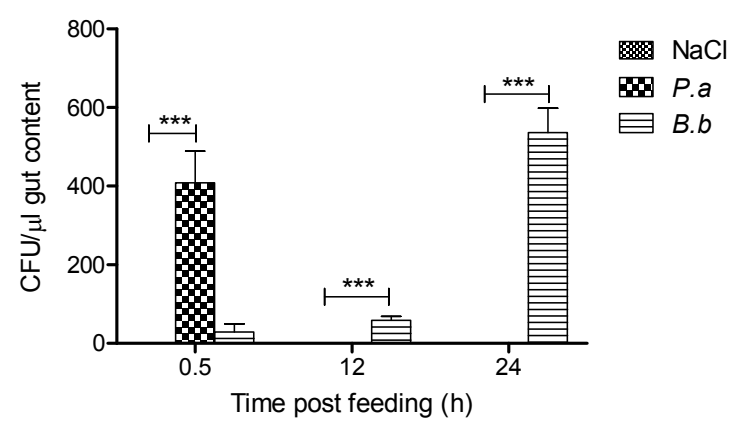

Fig. 2 CFU changes after the bacterial feeding NaCl: control; P.a: Pseudomonas aeruginosa; B.b: Bacillus bombyseptieus. Each value is given as the mean $\pm \mathrm{SD}$ of three replicates. ${ }^{*},{ }^{* *}$, and ${ }^{* * *}$ indicate statistical significance at $P<0.05, P<0.01$, and $P<0.001$, respectively. Three larvae were used at each time point, and the experiment was repeated three times 


\section{3 $\mathrm{H}_{2} \mathrm{O}_{2}$ levels in the gut and expression of genes involved in $\mathrm{H}_{2} \mathrm{O}_{2}$ metabolism after bacterial infection}

We next measured the $\mathrm{H}_{2} \mathrm{O}_{2}$ concentration in the gut after bacterial infection. Both $P$. aeruginosa and $B$. bombysepticus infection significantly increased intestinal $\mathrm{H}_{2} \mathrm{O}_{2}$ levels at 8 and $16 \mathrm{~h}$ (Fig. 3a). The Duox and catalase $(C A T)$ transcription levels were highly up-regulated by $P$. aeruginosa infection and downregulated by $B$. bombysepticus infection at $8 \mathrm{~h}$. At other post-infection times, the expression levels of these two genes were lower than those of the control (Figs. $3 \mathrm{~b}$ and $3 \mathrm{c}$ ). These data suggest that $\mathrm{H}_{2} \mathrm{O}_{2}$ is an important defense molecule in response to bacterial challenge.

\subsection{Changes in the level of NO in the gut and the transcription of genes related to NO metabolism after bacterial infection}

We also measured intestinal NO levels after bacterial infection. After $P$. aeruginosa infection, the NO concentration was lower than that of the control at $8 \mathrm{~h}$, followed by a significant increase at $16 \mathrm{~h}$ and then a decrease at $24 \mathrm{~h}$; whereas after $B$. bombysepticus infection, the NO level was slightly elevated only at 24 h (Fig. 4a). qPCR results showed that the NOS1 gene was down-regulated by both $P$. aeruginos $a$ and B. bombysepticus infection from 4 to $24 \mathrm{~h}$ (Fig. $4 \mathrm{~b}$ ). The NOS2 expression level was up-regulated at $8 \mathrm{~h}$ by $B$. bombysepticus infection and at $16 \mathrm{~h}$ by $P$. aeruginosa infection (Fig. 4c). These results imply that NO may play an important role in host defense and that NOS2 is involved in NO generation.

\subsection{Expression changes in AMP genes in response to bacterial challenge}

We measured the expression levels of six AMP genes (Attacin 2 (Att2), Cecropin B6 (CecB6), Cecropin D (CecD), Glovorin 2 (Glov2), Glovorin 3 (Glov3), and Morricin (Mor)) in the midgut using qPCR. All AMP genes were highly up-regulated from 4 to $24 \mathrm{~h}$ post-infection except for Mor (Fig. 5). Glov2 and Glov3 were the two most induced genes. At $4 \mathrm{~h}$ after $P$. aeruginosa and $B$. bombysepticus infection, the expression of Glov2 mRNA increased approximately 90 -fold compared with the control, while the transcription level of Glov3 induced 500- and 650-fold by $P$. aeruginosa and $B$. bombysepticus infection, (a)

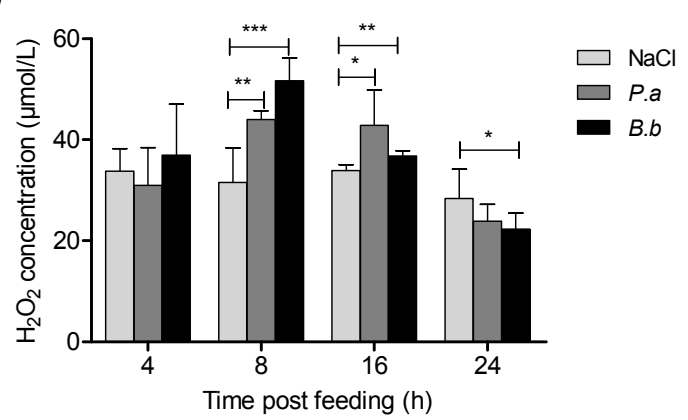

(b)

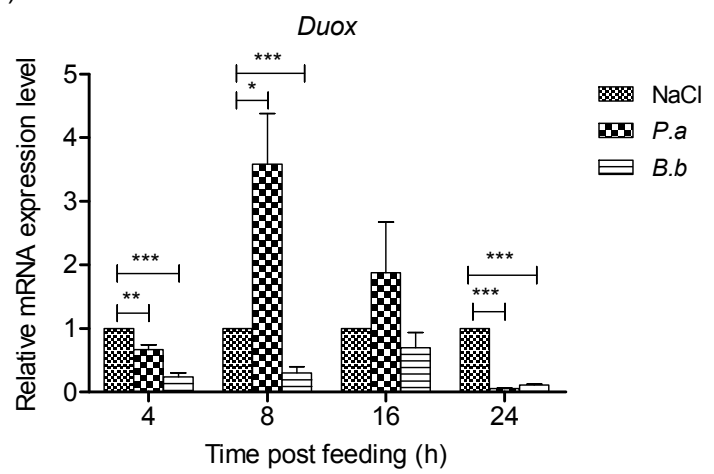

(c)

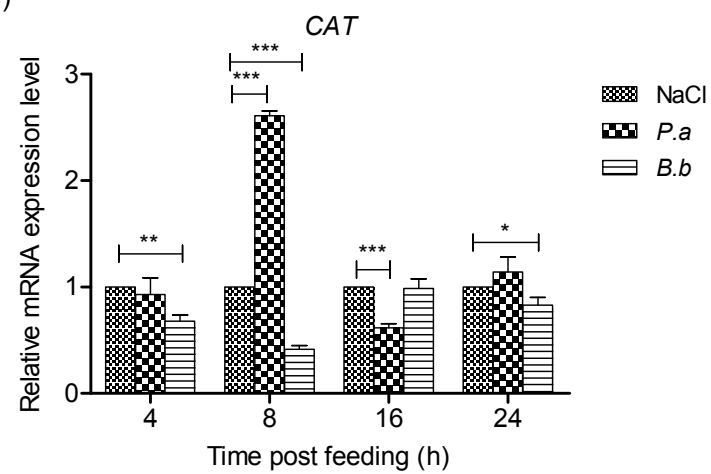

Fig. $3 \mathrm{H}_{2} \mathrm{O}_{2}$ concentrations (a) in the gut after bacterial feeding and Duox (b) and CAT (c) expression levels after bacterial feeding

In (b) and (c), shown are the relative expression levels of Duox and CAT compared to IF $4 \alpha$. NaCl: control; P.a: Pseudomonas aeruginosa; B.b: Bacillus bombyseptieus. Each value is given as the mean $\pm \mathrm{SD}$ of three replicates. ${ }^{*},{ }^{* *}$, and ${ }^{* * *}$ indicate statistical significance at $P<0.05, P<0.01$, and $P<0.001$, respectively. Three larvae were used at each time point, and the experiment was repeated three times

respectively. The transcription level profile of Mor differed from the other AMP genes. It was only significantly up-regulated at 8 and 24 h by $P$. aeruginosa infection. At other time points, both $P$. aeruginosa and $B$. bombysepticus infection caused a reduction in the Mor expression. At $24 \mathrm{~h}$, only Att2 and 
(a)

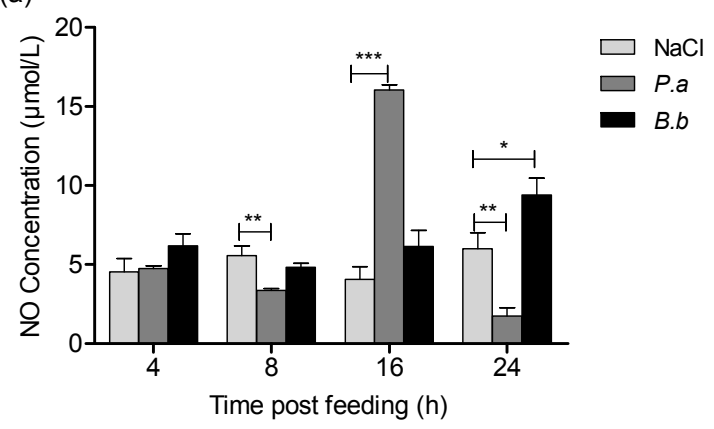

(b)

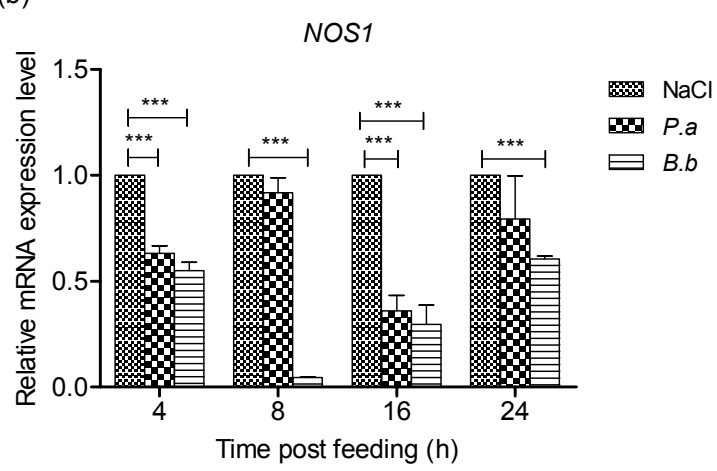

(c)

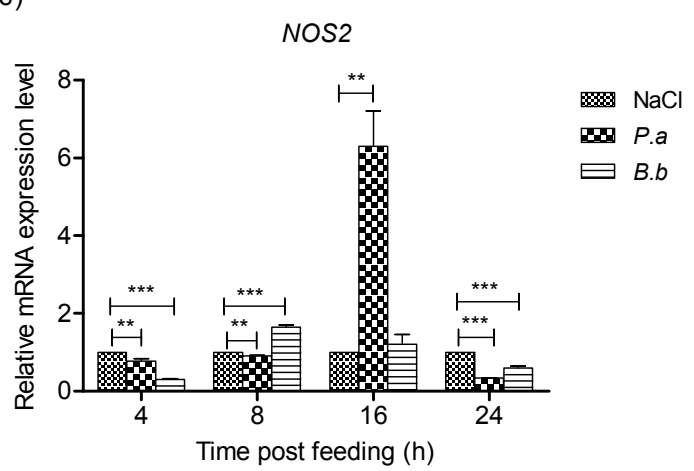

Fig. 4 NO concentrations (a) in the gut content after bacterial feeding and NOS1 (b) and NOS2 (c) expression levels after bacterial feeding

In (b) and (c), shown are the relative expression levels of NOS1 and NOS2 compared to IF $4 \alpha$. NaCl: control; P.a: Pseudomonas aeruginosa; B.b: Bacillus bombyseptieus. Each value is given as the mean $\pm \mathrm{SD}$ of three replicates. ${ }^{*},{ }^{* *}$, and ${ }^{* * *}$ indicate statistical significance at $P<0.05, P<0.01$, and $P<0.001$, respectively. Three larvae were used at each time point, and the experiment was repeated three times

Glov3 were induced at higher levels (15- and 100-fold, respectively) by $P$. aeruginosa and $B$. bombysepticus infection. These findings suggest that Glov2 and Glov3 are the major AMPs used to cope with early stage bacterial invasion.

\section{Discussion}

To defend against ingested harmful microorganisms, the insect gut has evolved an effective immune system depending on the local production of AMPs and ROS (Lemaitre and Hoffmann, 2007).

Drosophila showed high lethality under $P$. aeruginosa infection, which activated both the Toll and IMD pathways (Lau et al., 2003). P. aeruginosa exhibited higher pathogenicity to flies in the hemocoel than in the intestine (Chieda et al., 2005). B. bombysepticus, which produces spores and parasporal crystals, was reported to be a highly pathogenic bacterium in silkworms. B. bombysepticus oral infection could provoke strong host immune responses (Huang et al., 2009). In our study, we rarely found live $P$. aeruginosa in the gut at $12 \mathrm{~h}$ after oral infection (Fig. 2), which is likely caused by the orally invading $P$. aeruginosa being eliminated by the silkworm intestinal immune system; whereas the proliferation of B. bombysepticus increased gradually from 0.5 to $12 \mathrm{~h}$ (Fig. 2). The higher CFU of B. bombysepticus in the gut at $24 \mathrm{~h}$ is consistent with the higher silkworm mortality after infection (Fig. 1). Furthermore, our results showed that the expression of Duox and CAT was induced by $P$. aeruginosa, but not by $B$. bombysepticus (Figs. $3 \mathrm{~b}$ and $3 \mathrm{c}$ ), though both bacteria increased local ROS levels. We found that $B$. bombysepticus infection induced ROS production only at $8 \mathrm{~h}$ (Fig. 3a). Afterwards, ROS levels, Duox and CAT expression were barely increased after $8 \mathrm{~h}$ (Figs. $3 \mathrm{~b}$ and $3 \mathrm{c}$ ). We speculate that proliferation of $B$. bombysepticus is able to rapidly overcome the silkworm intestinal immune system and gradually cause epithelial cells damage, and finally block further ROS generation. The high level of ROS at $8 \mathrm{~h}$ might not be produced by the gut epithelia, but from the haemolymph instead.

In Drosophila, Attacin, Diptericin, Defensin, and Mechtnikowin genes were induced in the gut after Ecc15 oral infection (Buchon et al., 2009). In our study, among the six AMP genes induced in the midgut by $P$. aeruginosa and $B$. bombysepticus infection, Glov2 and Glov3 were significantly upregulated at $4 \mathrm{~h}$ (Fig. 5), while local ROS levels showed no changes between infected and control individuals (Fig. 3a). These results imply that Glov2 and Glov3 may serve as the main intestinal defense molecules during the early infection stage. 
(a)

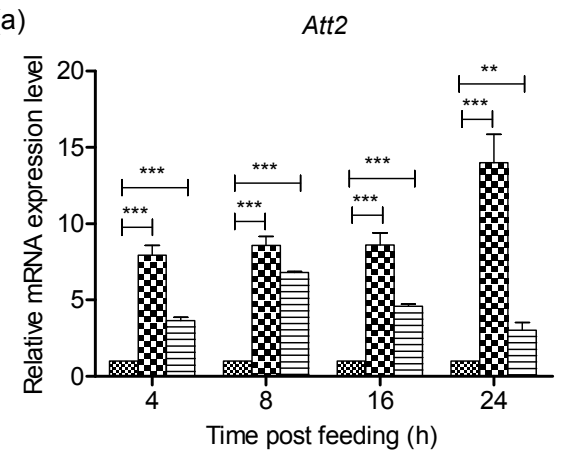

(c)

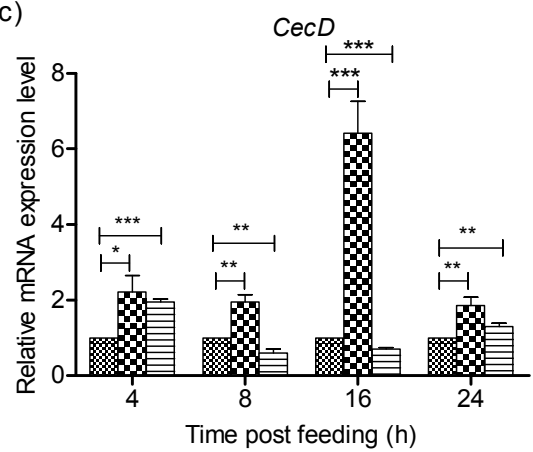

(e)

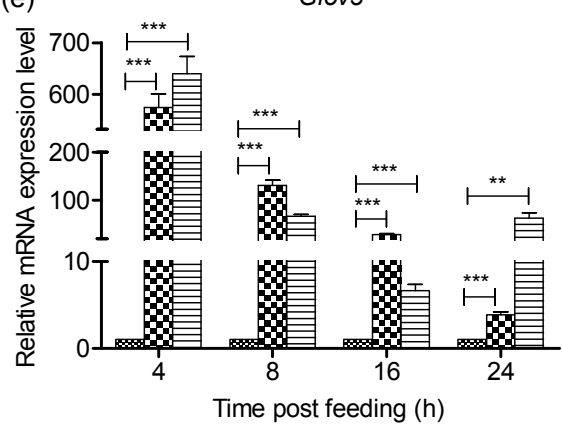

(b)

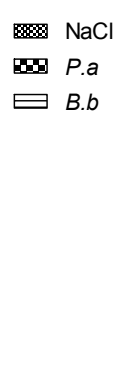

$\approx \mathrm{NaCl}$

P.a

$\sqsupseteq B . b$

(d)

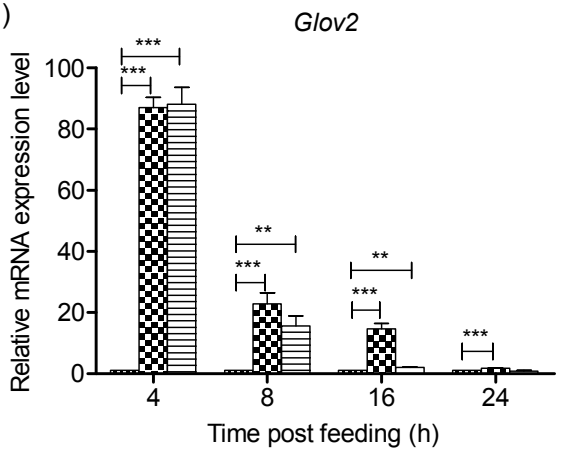

(f)

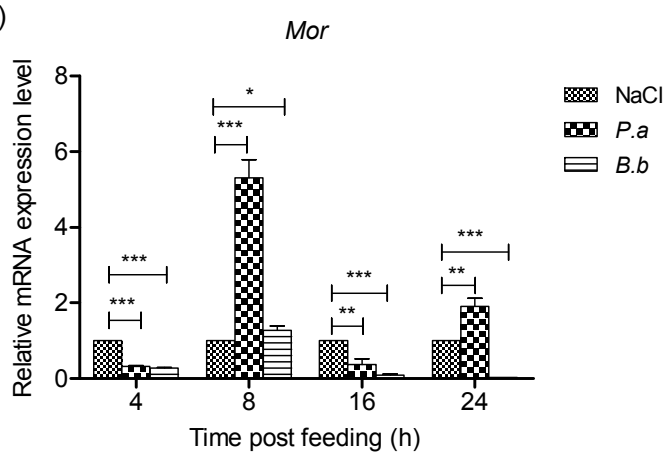

Fig. 5 mRNA expression levels of AMP genes after bacterial feeding

Shown are the relative expression levels of AMP genes in relation to $I F 4 \alpha$. Each value is given as the mean \pm SD of three replicates. ${ }^{*},{ }^{* *}$, and ${ }^{* * *}$ indicate statistical significance at $P<0.05, P<0.01$, and $P<0.001$, respectively. Three larvae were used at each time point, and the experiment was repeated three times

Natural gut infection also activates the IMD pathway to induce local AMP gene transcription, which plays complementary roles in combating ROS-resistant microbes (Ryu et al., 2006). In our study, at $24 \mathrm{~h}$, intestinal ROS levels were relatively low after $P$. aeruginosa and $B$. bombysepticus infection (Fig. 3a). However, the expression levels of AMP genes were still high. These results indicate that local AMP has more persistent antibacterial activity than ROS.
ROS signaling triggers local NO production by inducing intestinal NOS transcription in Drosophila (Wu et al., 2012). NO is induced and serves as an innate immune signal in response to gram-negative bacteria challenge (Foley and O'Farrell, 2003). NO is generated by inducible NOS2 (Wink et al., 2011). NO is thought to induce AMP gene expression by activating the IMD pathway in bacterially challenged Drosophila (Nappi et al., 2000). In the silkworm, Ceropin D was specifically activated by the IMD 
pathway after Escherichia coli and Bacillus subtilis infections (Tanaka et al., 2009). In our study, the expression of NOS2 was significantly induced by $P$. aeruginosa at $16 \mathrm{~h}$, leading to an increase of the NO level in the gut (Figs. 4a and 4c). It is notable that the transcription of $C e c D$ was highly up-regulated by $P$. aeruginosa infection at $16 \mathrm{~h}$ (Fig. 5c). It is possible that the elevated NO serves as a signal to induce $C e c D$ expression. Given the facts that there was no living $P$. aeruginosa in the gut after $12 \mathrm{~h}$ and the $\mathrm{H}_{2} \mathrm{O}_{2}$ level increased earlier than $\mathrm{NO}$ after bacterial challenge, we speculate that $\mathrm{H}_{2} \mathrm{O}_{2}$ induces $\mathrm{NO}$ generation in Bombyx as in Drosophila (Wu et al., 2012), though this hypothesis needs further investigation.

Taken together, we investigated the intestinal immune defenses after oral bacterial infection in the silkworm. Our results show that ROS and AMP play important roles in protecting the host against bacterial infection.

\section{Compliance with ethics guidelines}

Lei ZHANG, Yan-wen WANG, and Zhi-qiang LU declare that they have no conflict of interest.

All institutional and national guidelines for the care and use of laboratory animals were followed.

\section{References}

Basset, A., Khush, R.S., Braun, A., et al., 2000. The phytopathogenic bacteria, Erwinia carotovora, infects Drosophila and activates an immune response. PNAS, 97(7): 3376-3381. [doi:10.1073/pnas.97.7.3376]

Buchon, N., Broderick, N.A., Poidevin, M., et al., 2009. Drosophila melanogaster intestinal response to bacterial infection: activation of host defense and stem cell proliferation. Cell Host Microbe, 5(2):200-211. [doi:10.1016/ j.chom.2009.01.003]

Chieda, Y., Iiyama, K., Yasunaga-Aoki, C., et al., 2005. Pathogenicity of gacA mutant of Pseudomonas aeruginosa PA01 in the silkworm, Bombyx mori. FEMS Microbiol. Lett., 244(1):181-186. [doi:10.1016/j.femsle.2005. 01.032]

Foley, E., O'Farrell, P.H., 2003. Nitric oxide contributes to induction of innate immune responses to gram-negative bacteria in Drosophila. Genes Dev., 17(1):115-125. [doi:10.1101/gad.1018503]

Ha, E.M., Oh, C.T., Ryu, J.H., et al., 2005a. An antioxidant system required for host protection against gut infection in Drosophila. Dev. Cell, 8(1):125-132. [doi:10.1016/j. devcel.2004.11.007]

Ha, E.M., Oh, C.T., Bae, Y.S., et al., 2005b. A direct role for dual oxidase in Drosophila gut immunity. Science, 310(5749):847-850. [doi:10.1126/science.1117311]

Hoffmann, J.A., Reichhart, J.M., 2002. Drosophila innate immunity: an evolutionary perspective. Nat. Immunol., 3(2):121-126. [doi:10.1038/ni0202-121]

Huang, L., Cheng, T., Xu, P., et al., 2009. A genome-wide survey for host response of silkworm, Bombyx mori during pathogen Bacillus bombyseptieus infection. PLoS ONE, 4(12):e8098. [doi:10.1371/journal.pone.0008098]

Imamura, M., Yang, J., Yamakawa, M., 2002. cDNA cloning, characterization and gene expression of nitric oxide synthase from the silkworm, Bombyx mori. Insect Mol. Biol., 11(3):257-265. [doi:10.1046/j.1365-2583.2002.00333.x]

Lau, G.W., Goumnerov, B.C., Walendziewicz, C.L., et al., 2003. The Drosophila melanogaster toll pathway participates in resistance to infection by the gram-negative human pathogen Pseudomonas aeruginosa. Infect. Immun., 71(7):4059-4066. [doi:10.1128/IAI.71.7.4059-4066. 2003]

Lemaitre, B., Hoffmann, J., 2007. The host defense of Drosophila melanogaster. Annu. Rev. Immunol., 25(1):697743. [doi:10.1146/annurev.immunol.25.022106.141615]

Livak, K.J., Schmittgen, T.D., 2001. Analysis of relative gene expression data using real-time quantitative PCR and the $2^{-\Delta \Delta C_{\mathrm{T}}}$ method. Methods, 25(4):402-408. [doi:10.1006/ meth.2001.1262]

Nappi, A.J., Vass, E., Frey, F., et al., 2000. Nitric oxide involvement in Drosophila immunity. Nitric Oxide, 4(4): 423-430. [doi:10.1006/niox.2000.0294]

Oliveira, J.H.M., Gonçalves, R.L.S., Lara, F.A., et al., 2011. Blood meal-derived heme decreases ROS levels in the midgut of Aedes aegypti and allows proliferation of intestinal microbiota. PLoS Pathog., 7(3):e1001320. [doi:10.1371/journal.ppat.1001320]

Ryu, J.H., Ha, E.M., Oh, C.T., et al., 2006. An essential complementary role of NF- $\mathrm{KB}$ pathway to microbicidal oxidants in Drosophila gut immunity. EMBO J., 25(15): 3693-3701. [doi:10.1038/sj.emboj.7601233]

Tanaka, H., Sagisaka, A., Nakajima, Y., et al., 2009. Correlation of differential expression of silkworm antimicrobial peptide genes with different amounts of Rel family proteins and their gene transcriptional activity. Biosci. Biotechnol. Biochem., 73(3):599-606. [doi:10.1271/bbb.80685]

Tzou, P., Ohresser, S., Ferrandon, D., et al., 2000. Tissuespecific inducible expression of antimicrobial peptide genes in Drosophila surface epithelia. Immunity, 13(5): 737-748. [doi:10.1016/S1074-7613(00)00072-8]

Wink, D.A., Hines, H.B., Cheng, R.Y., et al., 2011. Nitric oxide and redox mechanisms in the immune response. $J$. Leukoc. Biol., 89(6):873-891. [doi:10.1189/jlb.1010550]

Wu, S., Zhang, X., Chen, X., et al., 2010a. BmToll9, an arthropod conservative Toll, is likely involved in the local gut immune response in the silkworm, Bombyx mori. Dev. Comp. Immunol., 34(2):93-96. [doi:10.1016/j.dci.2009. 08.010]

Wu, S., Zhang, X., He, Y., et al., 2010b. Expression of antimicrobial peptide genes in Bombyx mori gut modulated by oral bacterial infection and development. Dev. Comp. Immunol., 34(11):1191-1198. [doi:10.1016/j.dci.2010.06. 013] 
Wu, S.C., Liao, C.W., Pan, R.L., et al., 2012. Infectioninduced intestinal oxidative stress triggers organ-to-organ immunological communication in Drosophila. Cell Host Microbe, 11(4):410-417. [doi:10.1016/j.chom.2012.03.004]

\section{中文概要}

题 目：细菌感染引起的家蚕中肠免疫反应研究

目 的: 探索经喂食细菌感染引起的家蚕肠道内免疫反应 变化情况。

创新点：证明了家虫肠道内的活性氧（ROS）、一氧化氮 （NO）及抗菌肽在肠道免疫反应中的重要作用。

方 法: 通过绿脓杆菌 (Pseudomonas aeruginosa) 及黑胸 败血菌 (Bacillus bombysepticus) 喂食感染家蚕以 后，统计家蚕死亡率、检测感染后不同时间肠道 内过氧化氢 $\left(\mathrm{H}_{2} \mathrm{O}_{2}\right)$ 及 $\mathrm{NO}$ 的水平变化; 同时利用
实时苂光定量聚合酶链反应 (qPCR) 检测中肠组 织中活性氧相关基因及抗菌肽基因的转录情况。

结 论: 死亡率结果显示, 黑胸败血菌比绿脓杆菌具有更 强的致病性。活性氧检测结果显示, 喂食细菌感 后 $8 \mathrm{~h}$ 到 $16 \mathrm{~h}$, 家蚕肠道内 $\mathrm{H}_{2} \mathrm{O}_{2}$ 及 $\mathrm{NO}$ 水平显著升 高。通过 qPCR 研究 ROS 相关基因的表达变化的 结果显示, P. aeruginosa 感染后 $8 \mathrm{~h}$ 可诱导肠道 内双氧化酶 (Duox) 及过氧化氢酶 (CAT) 的转 录上调, 而感染后 $16 \mathrm{~h}$, P. aeruginosa 可诱导 NO 合成关键基因（一氧化氮核酶 2,NOS2) 的上调 表达, 喂食细菌感染同样可以诱导家䖯中肠抗菌 肽基因的上调表达, 而抗菌肽 Glovorin 2 及 Glovorin 3 在感染初期转录上调最为明显。实验 结果进一步证明 ROS、NO 及 AMP 的产生在家 虫肠道免疫防御中的重要作用。

关键词: 家虫; 中肠; 免疫; 过氧化氢; 一氧化氮; 抗菌 肽 